\title{
Fatores associados ao conhecimento de agentes comunitários de saúde em relação à saúde bucal
}

\author{
Factors associated with knowledge of community health \\ workers about oral health
}

\author{
Tatiane Brito Santana¹, Bruno Del Sarto Azevedo², \\ Ana Carolina Del-Sarto Azevedo Maia ${ }^{3}$
}

\begin{abstract}
Resumo
Introdução: É importante que os agentes comunitários de saúde (ACS) possuam conhecimentos suficientes para a realização de práticas educativas e o encaminhamento adequado do usuário, colaborando para o enfrentamento dos problemas de saúde pela população. Objetivo: Analisar os fatores associados ao conhecimento de ACS em relação à saúde bucal. Método: Estudo transversal, realizado com 255 ACS, em Jequié, Bahia, Brasil. Dois questionários foram utilizados para caracterização sociodemográfica e ocupacional e para avaliação do conhecimento sobre saúde bucal. A magnitude das associações foi aferida por meio da razão de prevalência e do intervalo de confiança de 95\%. Resultados: O baixo nível de conhecimento sobre saúde bucal se mostrou diretamente associado ao sexo masculino, à menor escolaridade, ao maior tempo de serviço na profissão e à ausência do cirurgião-dentista no quadro funcional da unidade. Conclusão: Os achados deste estudo fornecem novos conhecimentos a serem considerados por gestores e profissionais responsáveis pela educação em saúde bucal dos ACS, sobretudo ao destacar o impacto positivo que pode ter a presença do dentista dentro da equipe de saúde para a expansão desses conhecimentos.
\end{abstract}

Palavras-chave: agentes comunitários de saúde; saúde bucal; conhecimentos, atitudes e prática em saúde.

\begin{abstract}
Background: It is important that the community health workers ( $\mathrm{CHW}$ ) have enough knowledge to conduct educational practices and adequate referral of users, thus collaborating to address the health problems of the population. Objective: In this context, the objective of this study was to analyze the factors associated with knowledge of CHW about oral health. Method: This is a cross-sectional study conducted with $255 \mathrm{CHW}$ in the municipality of Jequié, Bahia state, Brazil. Two questionnaires were applied to $\mathrm{CHW}$ for sociodemographic and occupational characterization and evaluation of knowledge about oral health. Magnitude of association was assessed by prevalence ratio and 95\% confidence interval. Results: Low level of knowledge about oral health proved to be directly associated with male gender, lower education, longer service life as $\mathrm{CHW}$, and absence of a dentist in the staff of the health care unit. Conclusion: The findings of this study provide new knowledge to be considered by managers and professionals responsible for the education in oral health of $\mathrm{CHW}$, especially by highlighting the positive impact that the presence of a dentist in the health team may have on the expansion of this knowledge.

Keywords: community health workers; oral health; knowledge, attitudes, and practice in health.

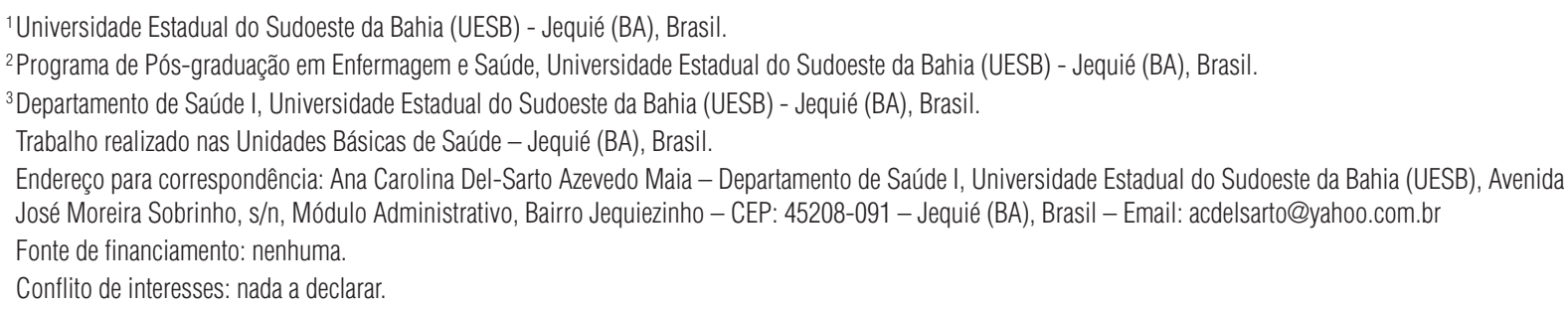




\section{INTRODUÇÃO}

Em razão do contato direto e constante com a população, os agentes comunitários de saúde (ACS) se configuram como um elo entre a comunidade e o sistema de saúde. Por essa maior proximidade, eles são capazes de reconhecer e apontar as necessidades das famílias adscritas no território, buscando, com a equipe de saúde, melhorias para a qualidade de vida e o bem-estar dessa coletividade'.

Além do desenvolvimento de atividades de promoção da saúde, de prevenção das doenças e dos agravos e de vigilância à saúde, por meio de visitas domiciliares e de ações educativas individuais e coletivas nos domicílios e na comunidade ${ }^{2}$, os ACS exercem ainda relevante papel na divulgação de informações sobre saúde bucal ${ }^{3}$.

No contexto da saúde bucal, os ACS oferecem mecanismos capazes de facilitar o desenvolvimento de ações que promovam benefícios para os usuários atendidos, por meio de ações de educação em saúde bucal, pelo agendamento de consultas odontológicas ou ainda pelo aumento da utilização dos serviços básicos de saúde, a fim de evitar a assistência odontológica tardia e reduzir a necessidade de consultas de urgência ${ }^{4,5}$. Dessa maneira, é essencial que os agentes possuam saberes suficientes para a realização de práticas educativas, além de estarem preparados para solucionar ou encaminhar quaisquer dúvidas sobre saúde bucal apresentadas pelos moradores da sua área de abrangência, colaborando para o enfrentamento dos problemas de saúde pela população $0^{6,7}$.

Considerando que a construção do conhecimento é um dos elementos que contribui para a população aumentar suas habilidades no controle dos determinantes e condicionantes do processo saúde-doença, bem como se tornar mais apta ao enfrentamento dos problemas de saúde bucal, faz-se necessário investigar os fatores que exercem influência sobre o maior ou o menor conhecimento em saúde bucal dos ACS, importantes atores sociais na divulgação de informações e de saberes. Tendo em vista, ainda, o fato de serem escassos os trabalhos que tiveram como objeto essa investigação, o objetivo deste estudo foi analisar os fatores associados ao conhecimento de ACS em relação à saúde bucal.

\section{MÉTODO}

\section{Desenho e população do estudo}

Trata-se de um estudo de corte transversal, de caráter censitário, realizado no município de Jequié, Bahia, que possuía uma população estimada, para o ano de 2015, de mais de 161 mil habitantes ${ }^{8}$.

Foram considerados como participantes elegíveis para este estudo todos os ACS em atividade nas zonas urbana e rural do município. Foram excluídos os agentes que se encontravam afastados do trabalho em função de férias ou licença (prêmio, saúde, gestação, interesse particular etc.). Os ACS foram abordados em seu ambiente de trabalho após contato prévio e agendamento com o(a) coordenador(a) de cada unidade. No caso dos agentes que não se fizeram presentes no dia da coleta de dados, uma nova tentativa de contato foi realizada no intuito de que fossem incluídos entre os participantes do estudo.

\section{Variáveis do estudo}

A coleta de dados se deu por meio de dois questionários, ambos autopreenchíveis. O primeiro foi empregado para caracterização sociodemográfica e ocupacional, formulado pelos pesquisadores responsáveis. Por meio dele, as seguintes variáveis foram investigadas: sexo, idade, situação conjugal, número de filhos, escolaridade, unidade de atuação, carga horária semanal de trabalho, tempo de serviço como agente, tipo de vínculo empregatício, salário líquido mensal como ACS, se possuía outro trabalho remunerado, presença e participação do cirurgião-dentista $(C D)$ na equipe, possibilidade de acesso ao dentista da unidade para obtenção de informação ou esclarecimento e realização/frequência com que era fornecida educação continuada sobre saúde bucal.

O segundo instrumento se refere ao questionário elaborado por Frazão e Marques ${ }^{9}$, composto por 21 questões e que possui as necessárias propriedades de validade de face e de conteúdo. Para a presente pesquisa, foram utilizadas 13 dessas questões, com o propósito de identificar os conhecimentos sobre saúde bucal em diferentes fases e condições de vida - infância, adolescência, vida adulta, gestação e terceira idade. Esse questionário é formado por questões de múltipla escolha, em que as perguntas apresentam categorias de respostas fixas para que o respondente escolha a que melhor representa a sua percepção de saúde bucal ${ }^{9}$.

Procedeu-se à categorização do nível de conhecimento sobre saúde bucal dos ACS por meio dos tercis da soma dos acertos dos participantes. Dessa forma, ficou categorizado como: nível de conhecimento baixo para aqueles agentes que acertaram 7 ou menos questões; nível de conhecimento moderado para aqueles que acertaram 8 ou 9 questões; e nível de conhecimento alto para aqueles que acertaram 10 ou mais questões.

\section{Análise estatística}

Utilizaram-se os programas EpiData 3.1 para a tabulação dos dados e SPSS 21.0 para a análise propriamente dita. As variáveis categóricas do estudo foram apresentadas por meio de frequências absolutas e relativas, enquanto as variáveis contínuas foram apresentadas por meio da média e do desvio-padrão.

A associação entre o conhecimento sobre saúde bucal (variável dependente) dos ACS - a partir da dicotomização das categorias previamente estabelecidas pelos tercis (níveis de conhecimento baixo e moderado-alto) - e as características sociodemográficas e ocupacionais (variáveis independentes) foi aferida por meio do teste do Qui-quadrado ou teste Exato de Fisher. Para a magnitude da associação, foi utilizada como medida de associação a razão de prevalência (RP) com respectivo intervalo de confiança (IC) de $95 \%$. Adotou-se o nível de significância estatística menor que 5\%. 


\section{Aspectos éticos}

O presente estudo foi aprovado pelo Comitê de Ética em Pesquisa da Universidade Estadual do Sudoeste da Bahia (CAAE: 28555814.5.0000.0055). Todos os participantes da pesquisa assinaram o Termo de Consentimento Livre e Esclarecido.

\section{RESULTADOS}

De um total de 345 ACS do quadro funcional do município, 22 se encontravam em gozo de férias, 19 estavam em licença por motivos médicos, 25 em licença-prêmio, e 3 em licença-gestação, o que totalizou 69 trabalhadores excluídos do estudo; assim, 276 agentes estavam elegíveis para participar da pesquisa. Contudo, houve 21 perdas, a saber: $16(5,8 \%)$ ACS com os quais não foi possível efetuar contato e $5(1,8 \%)$ que se recusaram a participar da pesquisa. Dessa forma, participaram efetivamente do estudo 255 agentes, o que equivale a uma taxa de resposta de $92,4 \%$

A maior parte dos ACS constituiu-se de indivíduos do sexo feminino (84,7\%), com média de idade de $42,7( \pm 9,1)$ anos, casados(as) ou com companheiros(as) (77,3\%), que possuíam dois filhos (31,6\%), com ensino médio completo (69\%) e uma média de tempo de serviço como agente de $12,9( \pm 4,9)$ anos (Tabela 1). Ressalta-se que todas as unidades da atenção primária à saúde do município foram representadas por pelo menos um agente entre os participantes da pesquisa.

Com relação às características laborais dos ACS do estudo (Tabela 2), 73,3\% deles atuavam na zona urbana, e $26,7 \%$, na zona rural. A maioria dos agentes mencionou possuir em sua unidade o CD dentro do quadro funcional $(64,4 \%)$, no qual esses profissionais participavam às vezes das reuniões de equipe (45,5\%). No que se refere à possibilidade de acesso dos agentes ao $\mathrm{CD}$ da unidade, a fim de obter qualquer informação ou esclarecimento, pouco mais da metade dos participantes $(53,5 \%)$ afirmou ter livre acesso ao profissional, não tendo qualquer dificuldade para interagir e dialogar com ele. No que concerne ao oferecimento de cursos de capacitação sobre saúde bucal, 73,5\% dos ACS relataram não existir tais capacitações. Dos $26,5 \%$ agentes que foram capacitados em algum momento, $77,4 \%$ deles informaram que as capacitações eram realizadas esporadicamente.

Todos os ACS possuíam vínculo efetivo (estatutário) e carga horária semanal de trabalho de 40 horas. Dos 237 agentes que informaram sua remuneração, 99,6\% relataram ganhar entre 1 e 2 salários mínimos (entre R $\$ 788,00$ e R \$ 1.576,00).

Dos 251 agentes que responderam a todas as 13 questões do questionário sobre saúde bucal, 87 (34,7\%) obtiveram um baixo nível de conhecimento, 81 (32,3\%), um moderado nível de conhecimento, e $83(33,1 \%)$, um alto nível de conhecimento em saúde bucal.
Tabela 1. Características sociodemográficas dos agentes comunitários de saúde, Jequié, 2015

\begin{tabular}{lcc}
\multicolumn{1}{c}{ Variáveis/categorias } & $\mathbf{n}$ & $\mathbf{\%}$ \\
$\begin{array}{l}\text { Sexo }(\mathbf{n}=\mathbf{2 5 5})^{\mathrm{a}} \\
\text { Masculino }\end{array}$ & 39 & 15,3 \\
Feminino & 216 & 84,7 \\
Faixa etária $(\mathbf{n}=\mathbf{2 3 9})^{\mathrm{a}}$ & & \\
20 a 29 anos & 13 & 5,4 \\
30 a 39 anos & 77 & 32,2 \\
40 a 49 anos & 95 & 39,7 \\
50 a 59 anos & 43 & 18,0 \\
$\geq 60$ anos & 11 & 4,6 \\
Situação conjugal $(\mathbf{n}=\mathbf{2 5 1})^{\mathrm{a}}$ & & \\
Solteiro(a) ou sem companheiro(a) & 57 & 22,7 \\
Casado(a) ou com companheiro(a) & 194 & 77,3 \\
Número de filhos $(\mathbf{n}=\mathbf{2 5 3})^{\mathbf{a}}$ & & \\
0 & 35 & 13,8 \\
1 & 65 & 25,7 \\
2 & 80 & 31,6 \\
$\geq 3$ & 73 & 28,9 \\
Escolaridade $(\mathbf{n}=\mathbf{2 5 5})^{\mathrm{a}}$ & & \\
Ensino fundamental & 26 & 10,2 \\
Ensino médio & 176 & 69,0 \\
Ensino superior & 53 & 20,8 \\
Tempo como ACS $(\mathbf{n}=\mathbf{2 5 5})^{\mathrm{a}}$ & & \\
$\leq 5$ anos & 4 & 1,6 \\
6 a 10 anos & 107 & 42,0 \\
11 a 15 anos & 35 & 13,7 \\
$\geq 16$ & 109 & 42,7 \\
\hline
\end{tabular}

${ }^{\mathrm{a}} \mathrm{O}$ número de respostas de cada variável varia em função das informações ignoradas

Tabela 2. Características laborais dos agentes comunitários de saúde, Jequié, 2015

\begin{tabular}{|c|c|c|}
\hline Variáveis/categorias & $\mathbf{n}$ & $\%$ \\
\hline \multicolumn{3}{|l|}{ Zona da unidade $(\mathbf{n}=255)^{\mathrm{a}}$} \\
\hline Zona urbana & 187 & 73,3 \\
\hline Zona rural & 68 & 26,7 \\
\hline \multicolumn{3}{|c|}{ Unidade com dentista na equipe $(n=250)^{a}$} \\
\hline $\operatorname{Sim}$ & 161 & 64,4 \\
\hline Não & 89 & 35,6 \\
\hline \multicolumn{3}{|c|}{$\begin{array}{l}\text { Participação do dentista nas reuniões de equipe } \\
\text { da unidade }(\mathrm{n}=154)^{\mathrm{a}}\end{array}$} \\
\hline Nunca & 63 & 40,9 \\
\hline Às vezes & 70 & 45,5 \\
\hline Sempre & 21 & 13,6 \\
\hline \multicolumn{3}{|c|}{$\begin{array}{l}\text { Livre acesso ao dentista da unidade para obter } \\
\text { qualquer informação ou esclarecimento }(n=157)^{a}\end{array}$} \\
\hline $\operatorname{Sim}$ & 84 & 53,5 \\
\hline Não & 73 & 46,5 \\
\hline \multicolumn{3}{|c|}{$\begin{array}{l}\text { Oferta de cursos de capacitação sobre saúde } \\
\text { bucal }(n=238)^{\mathrm{a}}\end{array}$} \\
\hline $\operatorname{Sim}$ & 63 & 26,5 \\
\hline Não & 175 & 73,5 \\
\hline \multicolumn{3}{|c|}{$\begin{array}{l}\text { Frequência da oferta de cursos de capacitação } \\
\text { sobre saúde bucal }(n=62)^{\mathrm{a}}\end{array}$} \\
\hline Pelo menos a cada seis meses & 1 & 1,6 \\
\hline Pelo menos uma vez ao ano & 13 & 21,0 \\
\hline Esporadicamente & 48 & 77,4 \\
\hline \multicolumn{3}{|c|}{ Outro emprego ou trabalho remunerado $(n=236)^{a}$} \\
\hline Sim & 5 & 2,1 \\
\hline Não & 231 & 97,9 \\
\hline
\end{tabular}

${ }^{2} \mathrm{O}$ número de respostas de cada variável varia em função das informações ignoradas 
Conforme a Tabela 3, entre as características sociodemográficas dos ACS, o baixo nível de conhecimento sobre saúde bucal se mostrou diretamente associado ao sexo masculino $(\mathrm{RP}=1,67$; IC95\%: 1,17-2,40), à menor escolaridade $(\mathrm{RP}=1,93$; IC95\%: 1,07-3,48, para quem possuía ensino médio completo; $\mathrm{RP}=2,16$; IC95\%: 1,06-4,41, para quem possuía ensino fundamental completo) e ao maior tempo de serviço como ACS (RP = 1,56; IC95\%: 1,08-2,26).

Entre as características laborais dos ACS presentes na Tabela 4, o baixo nível de conhecimento sobre saúde bucal se mostrou diretamente associado à ausência do dentista no quadro funcional da unidade ( $\mathrm{RP}=1,46$; IC95\%: 1,05-2,05).

Tabela 3. Prevalência (P\%), razões de prevalência (RP) e intervalos de confiança (IC95\%) de baixo nível de conhecimento sobre saúde bucal, segundo as características sociodemográficas entre agentes comunitários de saúde, Jequié, 2015

\begin{tabular}{|c|c|c|c|}
\hline Variáveis/categorias & $(\mathbf{P} \%)$ & $\mathrm{RP}\left(\mathrm{IC}_{95 \%}\right)$ & Valor de $p$ \\
\hline \multicolumn{4}{|l|}{ Sexo } \\
\hline Feminino & 31,5 & 1,00 & \\
\hline Masculino & 52,6 & $1,67(1,17-2,40)$ & 0,012 \\
\hline \multicolumn{4}{|l|}{ Faixa etária } \\
\hline 20 a 29 anos & 23,1 & 1,00 & \\
\hline 30 a 39 anos & 28,9 & $1,25(0,44-3,60)$ & 1,000 \\
\hline 40 a 49 anos & 34,0 & $1,47(0,53-4,14)$ & 0,539 \\
\hline$\geq 50$ anos & 46,2 & $2,00(0,71-5,63)$ & 0,209 \\
\hline \multicolumn{4}{|l|}{ Situação conjugal } \\
\hline Solteiro(a) ou sem companheiro(a) & 30,9 & 1,00 & \\
\hline Casado(a) ou com companheiro(a) & 34,9 & $1,13(0,73-1,75)$ & 0,582 \\
\hline \multicolumn{4}{|l|}{ Número de filhos } \\
\hline 0 & 29,4 & 1,00 & \\
\hline 1 & 29,7 & $1,01(0,53-1,92)$ & 0,977 \\
\hline 2 & 25,6 & $0,87(0,46-1,66)$ & 0,679 \\
\hline$\geq 3$ & 49,3 & $1,67(0,95-2,97)$ & 0,053 \\
\hline \multicolumn{4}{|l|}{ Escolaridade } \\
\hline Ensino superior & 19,6 & 1,00 & \\
\hline Ensino médio & 37,9 & $1,93(1,07-3,48)$ & 0,015 \\
\hline Ensino fundamental & 42,3 & $2,16(1,06-4,41)$ & 0,034 \\
\hline \multicolumn{4}{|l|}{ Tempo como ACS } \\
\hline$\leq 10$ anos & 26,4 & 1,00 & \\
\hline$>10$ anos & 41,1 & $1,56(1,08-2,26)$ & 0,015 \\
\hline
\end{tabular}

Tabela 4. Prevalência (P\%), razões de prevalência (RP) e intervalos de confiança (IC95\%) de baixo nível de conhecimento sobre saúde bucal, segundo as características laborais entre agentes comunitários de saúde, Jequié, 2015

\begin{tabular}{|c|c|c|c|}
\hline Variáveis/categorias & $(\mathbf{P} \%)$ & $\mathbf{R P}\left(\mathrm{IC}_{95 \%}\right)$ & Valor de $\mathbf{p}$ \\
\hline \multicolumn{4}{|l|}{ Zona da unidade } \\
\hline Zona urbana & 32,2 & 1,00 & \\
\hline Zona rural & 41,2 & $1,28(0,90-1,82)$ & 0,186 \\
\hline \multicolumn{4}{|l|}{ Unidade com dentista na equipe } \\
\hline Sim & 29,9 & 1,00 & \\
\hline Não & 43,8 & $1,46(1,05-2,05)$ & 0,028 \\
\hline \multicolumn{4}{|c|}{ Participação do dentista nas reuniões de equipe da unidade } \\
\hline Sempre & 23,8 & 1,00 & \\
\hline Às vezes & 27,5 & $1,16(0,49-2,72)$ & 0,735 \\
\hline Nunca & 35,0 & $1,47(0,63-3,40)$ & 0,344 \\
\hline \multicolumn{4}{|c|}{$\begin{array}{l}\text { Livre acesso ao dentista da unidade para obter qualquer } \\
\text { informação ou esclarecimento }\end{array}$} \\
\hline $\operatorname{Sim}$ & 26,2 & 1,00 & \\
\hline Não & 34,8 & $1,33(0,82-2,15)$ & 0,249 \\
\hline \multicolumn{4}{|c|}{ Oferta de cursos de capacitação sobre saúde bucal } \\
\hline Sim & 25,4 & 1,00 & \\
\hline Não & 34,5 & $1,36(0,85-2,18)$ & 0,186 \\
\hline \multicolumn{4}{|l|}{ Outro emprego ou trabalho remunerado } \\
\hline Não & 31,7 & 1,00 & \\
\hline Sim & 40,0 & $1,26(0,42-3,75)$ & 0,655 \\
\hline
\end{tabular}




\section{DISCUSSÃO}

Os resultados do presente estudo apontaram para um maior nível de conhecimento sobre saúde bucal entre as ACS do sexo feminino, característica essa potencialmente favorável para a realização das atividades inerentes à profissão, uma vez que as mulheres, geralmente, possuem maior sensibilidade para compreender e se responsabilizar pelos dilemas da comunidade, sendo consideradas "cuidadoras" na sociedade ${ }^{10}$.

A despeito da predominância de agentes com nível médio de ensino, ressalta-se o fato de que $20,8 \%$ desses indivíduos declararam ter concluído o ensino superior. Neste estudo, o grau de escolaridade influenciou nos conhecimentos sobre saúde bucal. Assim, quanto maior o grau de escolaridade dos ACS, maiores as condições para apreender novos conhecimentos, tornando possível uma melhor capacitação para orientar, de forma mais eficaz, as famílias sobre a responsabilidade e o autocuidado com a saúde ${ }^{1,10,11}$. O maior nível de escolaridade pode refletir positivamente também nos processos interacionais com a equipe e a comunidade, bem como na ampliação da criticidade e da criatividade dos ACS ${ }^{12}$.

O presente estudo demonstrou um menor nível de conhecimento sobre saúde bucal entre os ACS com maior tempo de serviço na profissão, o que pode ter relação direta com a maior idade do participante. Os ACS mais velhos tendem a ter seus conceitos sobre o processo saúde-doença definidos, podendo ser resistentes a novos conceitos sobre a promoção de saúde em sua área de abrangência. Em contrapartida, em se tratando dos agentes mais jovens, os conceitos de saúde e de doença podem não ser tão enraizados, por isso esses ACS são mais aptos a aceitar as mudanças e as novidades ${ }^{11}$.

Constatou-se que a presença do CD na equipe de saúde contribuiu de forma significativa para um maior nível de conhecimento sobre saúde bucal dos ACS, uma vez que o dentista, fazendo-se presente nas unidades, pode esclarecer as dúvidas, favorecendo a desmonopolização do conhecimento odontológico e levando-o até os lares das famílias. Com isso, colabora-se para o aumento da autonomia da população e sua corresponsabilidade na preservação e na promoção das condições de saúde bucal, além de possibilitar ações mais efetivas e eficientes, baseadas no vínculo, ${ }^{9,10,13,14}$. A literatura assinala ainda que a atuação de equipes de saúde bucal pode refletir em mudanças nas condições da saúde bucal da população, uma vez que é possibilitado que os ACS ampliem seus conhecimentos para a identificação dos problemas dos usuários, permitindo o encaminhamento adequado destes ${ }^{5,10}$.

Faz-se necessário que o CD esteja totalmente inserido no cotidiano e na rotina das unidades básicas, para que não apenas a população, mas a equipe e o sistema de saúde o reconheçam como parte fundamental do processo, saindo do isolamento e permitindo a troca de conhecimento com todos os integrantes da equipe, visando, assim, à interdisciplinaridade ${ }^{15,16}$. Para tanto, é essencial que o $\mathrm{CD}$ esteja disponível para o diálogo, que extrapole os muros da unidade, não ficando restrito ao seu consultório, voltando suas ações para a coletividade - sem detrimento das ações de caráter individual - e que as atividades de educação em saúde para a população e para os membros da equipe estejam arraigadas ao seu processo de trabalho.

A mudança ao longo dos últimos anos do perfil curricular dos cursos de odontologia faz nascer a perspectiva de um CD com uma formação acadêmica mais alicerçada na saúde coletiva. Essa é uma mudança, acima de tudo, necessária, uma vez que o CD teve, ao longo do tempo, sua formação embasada no emprego de tecnologias importadas, contrariando a realidade social brasileira ${ }^{17}$.

Vale acrescentar que algumas limitações se fazem presentes neste estudo. Entre elas, destaca-se o delineamento transversal, o que não permite inferências sobre a causalidade das associações observadas. Outra limitação diz respeito à inexistência de um ponto de corte sugestivo de melhor ou pior conhecimento sobre saúde bucal para o instrumento utilizado, o que inviabiliza comparações mais precisas e uniformes.

Em suma, o baixo nível de conhecimento sobre saúde bucal se mostrou diretamente associado ao sexo masculino, à menor escolaridade, ao maior tempo de serviço na ocupação e à ausência do dentista no quadro funcional da unidade.

Os achados deste estudo podem fornecer subsídio à tomada de decisão de gestores e profissionais responsáveis pela educação em saúde bucal dos ACS. Destacou-se ainda a importância que pode ter a presença e a participação ativa do CD dentro da equipe de saúde como fatores contributivos para expandir os conhecimentos sobre saúde bucal dos ACS e, dessa forma, promover uma ação efetiva deles como agentes capazes de impactar positivamente no processo saúde-doença da comunidade em que estão inseridos, possibilitando ao usuário mudar hábitos, apoiando-se na conquista de sua autonomia.

\section{REFERÊNCIAS}

1. Galavote HS, Prado TN, Maciel EL, Cássia Duarte Lima R. Desvendando os processos de trabalho do agente comunitário de saúde nos cenários revelados na Estratégia Saúde da Família no município de Vitória (ES, Brasil). Cien Saude Colet. 2011;16(1):231-40. http://dx.doi.org/10.1590/ S1413-81232011000100026. PMid:21180831.
2. Brasil. Ministério da Saúde. Portaria MS/GM no 2.488 de 21 de outubro de 2011. Aprova a Política Nacional de Atenção Básica, estabelecendo a revisão de diretrizes e normas para a organização da Atenção Básica, para a Estratégia Saúde da Família (ESF) e o Programa de Agentes Comunitários de Saúde (PACS). Diário Oficial da União, Brasília, 24 de outubro de 2011. 
3. Brasil. Ministério da Saúde. Departamento de Atenção Básica. Saúde Bucal: Cadernos de Atenção Básica, n 17. Brasília: Ministério da Saúde; 2008. (Série A. Normas e Manuais Técnicos).

4. Marzari CK, Junges JR, Selli L. Agentes comunitários de saúde: perfil e formação. Cien Saude Colet. 2011;16(Supl Suppl 1):873-80. http://dx.doi. org/10.1590/S1413-81232011000700019. PMid:21503435.

5. Frazão P, Marques DSC. Efetividade de programa de agentes comunitários na promoção de saúde bucal. Rev Saude Publica. 2009;43(3):463-71. http:// dx.doi.org/10.1590/S0034-89102009000300010.

6. Moretti-Pires RO, Lima LAM, Machado MH. Sociologia das profissões e percepção de acadêmicos de Odontologia sobre o agente comunitário de saúde em saúde bucal. Interface Comum Saude Educ. 2011;15(39):1085-96.

7. Santos KT, Saliba NA, Moimaz SA, Arcieri RM, Carvalho ML. Agente comunitário de saúde: perfil adequado a realidade do programa Saúde da Família? Cien Saude Colet. 2011;16(Supl Suppl 1):1023-8. http://dx.doi. org/10.1590/S1413-81232011000700035. PMid:21503451.

8. Brasil. Instituto Brasileiro de Geografia e Estatística. Diretoria de Pesquisas. Coordenação de População e Indicadores Sociais. Estimativa da População 2015 [Internet]. 2015; [citado 2015 set 20]. Disponível em: http://cod.ibge. gov.br/228

9. Frazão P, Marques DSC. Influência de agentes comunitários de saúde na percepção de mulheres e mães sobre conhecimentos de saúde bucal. Cien Saude Colet. 2006;11(1):131-44. http://dx.doi.org/10.1590/S141381232006000100021 .

10. Moura MS, Carvalho CJ, Amorim JTC, Marques MFSS, Moura LFAD, Mendes RF. Perfil e práticas de saúde bucal do agente comunitário de saúde em municípios piauienses de pequeno porte. Cien Saude Colet.
2010;15(Supl 1):1487-95. http://dx.doi.org/10.1590/S1413-81232010000700061. PMid:20640311.

11. Ferraz L, Aerts DR. O cotidiano de trabalho do agente comunitário de saúde no PSF em Porto Alegre. Cien Saude Colet. 2005;10(2):347-55. http://dx.doi.org/10.1590/S1413-81232005000200012.

12. Lino MM, Lanzoni GMM, Albuquerque GL, Schveitzer MC. Perfil socioeconômico, demográfico e de trabalho dos agentes comunitários de saúde. Cogitare Enferm. 2012;17(1):57-64. http://dx.doi.org/10.5380/ ce.v17i1.26375.

13. Rodrigues AAAO, Santos AM, Assis MMA. Agentes comunitários de saúde: sujeito da prática em saúde bucal em Alagoinhas, Bahia. Cien Saude Colet. 2010;15(3):907-15. http://dx.doi.org/10.1590/S1413-81232010000300034. PMid:20464204.

14. Mialhe FL, Lefévre F, Lefévre AMC. O agente comunitário de saúde e suas práticas educativas em saúde bucal: uma avaliação qualiquantitativa. Cien Saude Colet. 2011;16(11):4425-32. http://dx.doi.org/10.1590/S141381232011001200015. PMid:22124823.

15. Farias MR, Sampaio JJC. Papel do cirurgião-dentista na equipe de saúde da família. RGO. 2011;59(1):109-15.

16. Reis WG, Scherer MDA, Carcereri DL. O trabalho do Cirurgião-Dentista na Atenção Primária à Saúde: entre o prescrito e o real. Saúde Debate. 2015;39(104):56-64. http://dx.doi.org/10.1590/0103-110420151040608.

17. Narvai PC. Odontologia e saúde bucal coletiva. São Paulo: Hucitec; 1994.

Recebido em: Nov. 17, 2017 Aprovado em: Ago. 01, 2018 